STRUCTURAL BIOLOGY

\section{Iron-Sulfur Clusters in Chemistry and Biology. Volume 2: Biochemistry, Biosynthesis and Human Diseases. Edited by Tracey Rouault. De Gruyter, 2017. Pp. xxi + 470. Price EUR 99.95, GBP 90.99, USD 140.00, hardcover, ISBN 978-3-11-047985-0.}

\author{
Jean-Pierre Jacquot*
}

Université de Lorraine, INRA, Laboratoire Interaction Arbres Microorganismes, Nancy, France. *Correspondence e-mail: j2p@univ-lorraine.fr

Keywords: book reviews; iron-sulfur clusters; biochemistry, biosynthesis and human diseases.

The second volume of the second edition of the book entitled Iron-Sulfur Clusters in Chemistry and Biology, edited by Tracey Rouault and published by de Gruyter, addresses primarily the biochemical properties of iron-sulfur centres (ISCs), their biosynthesis and also, importantly, the dire consequences of some mutations for human diseases. The earlier edition of this book was coordinated by the same editor and the new version has been split into two different volumes. The first one deals primarily with physico-chemical and spectroscopic properties of iron-sulfur centres and iron-sulfur proteins, and it has been subject to an earlier evaluation by the same author (Jacquot, 2017). The second volume, analysed in the present review, contains 17 different chapters, four of them being new additions. A short preface by Tracy Rouault introduces the whole volume. Chapter 1 by Dos Santos and Dean retraces the discovery of the nif operon in Azobacter vinelandii, a nitrogen-fixing species, and the assembly of the metallic cofactors in nitrogenase. The structural features of these metallic centres have been largely discussed in Volume 1. Chapter 2 is a new addition by Barras and colleagues which details the ISC system of bacteria. The third system for ISC incorporation, the suf operon, is described in a third chapter by Wayne Outten who addresses the interesting question as to why suf and ISC coexist in some species such as Escherichia coli. The hypothesis that they are not equally sensitive to stress is obviously of high interest. The next chapter by Mettert et al. describes the properties and phylogeny of E. coli IscR, a cellular sensor of ISC demand, and its binding properties to DNA promoter regions. Chapter 5 by Dos Santos investigates the ISC assembly in Gram-positive bacteria which possess only one assembly system, i.e. clostridia (ISC), actinomycetes (SUF) and bacilli (SUF). In general, it seems that Grampositive bacteria contain fewer client proteins with iron-sulfur centres than their Gramnegative counterparts. Chapter 6 by Pain and Dancis transports us into the eukaryotic world, describing ISC assembly in yeast, essentially via the mitochondrial system. The next chapter by Caryn Outten describes the role of ISCs in the regulation of iron homeostasis in yeast. Moving further into the eukaryotic world, Tracey Rouault details the biogenesis of Fe-S proteins in mammals and the regulatory switches IRP1 and IRP2. This chapter is followed by a new addition by Maio and Rouault dealing with the delivery of ISCs to recipient proteins, detailing in particular the role of chaperone and cochaperone proteins and the importance of the LYR motif in target proteins for the binding to chaperones such as HSC20. Chapter 10 is the largest of them all by Wing Hang Tong with a whopping 600+ references. This analysis details a number of mutations either in ISC-containing proteins or in components of the assembly systems that lead to diseases with severe phenotypes. Table 10.1 of this chapter is very informative about the large number and variety of syndromes associated with mutations of these systems. The next chapter, also an innovation in this volume by Knight and Wilson concentrates on one of these diseases Friedreich ataxia, which results from a defect in frataxin, a protein essential for ISC synthesis and assembly. Then Silke Leimkühler discusses the connections between molybdenum cofactor biosynthesis, ISCs and tRNA thiolation in humans. Basically these reactions rely on cysteine desulfurases of the NFS type and on the formation of persulfides. In Chapter 13 Kerstin Gari draws our attention to the relationship between ISC-containing proteins and genome stability. The bottom line is that 
several restriction enzymes, primases, helicases and polymerases actually contain ISCs of various forms, and hence their activities are conditioned by the presence of these prosthetic groups. In Chapter 14, Roland Lill and colleagues pay extensive attention to the cytosolic assembly machinery CIA. Chapter 15 by Jacqueline Barton and colleagues is the last new contribution. It details DNA signalling by ISCcontaining proteins and, in several aspects, appears to be complementary to Chapter 13 by Gari. The penultimate chapter by Hong Ye describes iron-sulfur assembly in plants which possess, in addition to the mitochondrial and cytosolic systems, a third component in chloroplasts or plastids. Plants are fascinating organisms which unlike other eukaryotes are autotrophic for carbon, nitrogen and sulfur, and possess additional ISC-containing proteins needed for photosynthesis and several other anabolic pathways. Actually this chapter might have been better positioned after the one dealing with yeast and before those concerning mammalian systems and human diseases. Finally, the last chapter is a very fascinating and exciting one which explores various scenarios for the origin of life including the RNA-first theory, the protein-first theory, and also gives extreme credibility to a third possibility centring on the formation of inorganic ISCs near marine vents in areas of volcanic activity. It is hypothesized that ISCs have acted as primary catalysts for the development of life and that they were subsequently engulfed in protein bodies with optimized biological activities.

Compared with the earlier edition there has been a large effort towards modernisation, especially through the introduction of the new chapters. One example can be found in the large number of references pertaining to the involvement of glutaredoxins in ISC assembly that are made in various chapters of the present edition. This field is indeed expanding rapidly after the pioneering work published nearly a decade ago in two seminal papers by Lillig et al. (2005) on humans and Rouhier et al. (2007) on plants, who demonstrated that several Grxs bind ISCs with glutathione as ligands and readily exchange them to client proteins.

As can be guessed from this review I have enjoyed reading this volume enormously. I believe that it is well balanced and there is very little redundancy between the different chapters, which nicely complement one another. In this respect one should congratulate the overall editor Tracey Rouault. I think it is a great contribution to the field of iron-sulfur proteins and a great tool for students to understand the broad significance of these reactions in biology. I strongly recommend that all groups interested in this field purchase it together with the first volume, it is a very good investment. A short word for the crystallographers reading Acta Crystallographica Section D, it is full of protein structures, you will not be disappointed!

\section{References}

Jacquot, J.-P. (2017). Acta Cryst. D73, 1030.

Lillig, C. H., Berndt, C., Vergnolle, O., Lönn, M. E., Hudemann, C., Bill, E. \& Holmgren, A. (2005). Proc. Natl Acad. Sci. USA, 102, 8168-8173.

Rouhier, R., Unno, H., Bandyopadhyay, S., Masip, L., Kim, S.-K., Hirasawa, M., Gualberto, J. M., Lattard, V., Kusunoki, M., Knaff, D. B., Georgiou, G., Hase, T., Johnson, M. K. \& Jacquot, J. P. (2007). Proc. Natl Acad. Sci. USA, 104, 7379-7384. 